the flecnode curves. Combining this result with the theorem of the Tôhoku paper we have the following theorem.

Theorem. The planes osculating the flecnode curve, the complex curve and the harmonic curve at the six points in which these curves cut a line element of their supporting ruled surface, will belong to a pencil, if, and only if,

$$
D \equiv \stackrel{2}{p_{21} \Delta_{1}}-\stackrel{2}{p_{12} \Delta_{2}}=0 \text {. }
$$

Since, when $\Delta_{1}=\Delta_{2}=0$, the flecnode curves are plane, the theorem of Fubini-Čech appears as a special case of the preceding theorem.

The University of Washington

\title{
NETS OF CONICS IN THE GALOIS FIELDS OF ORDER $2^{n *}$
}

\section{BY A. D. CAMPBELL}

We consider a net of conics in the Galois fields of order $2^{n}$ (1)

$$
\lambda C_{1}+\mu C_{2}+\nu C_{3}=0,
$$

where

$C_{i} \equiv a_{i} x^{2}+b_{i} y^{2}+c_{i} z^{2}+f_{i} y z+g_{i} z x+h_{i} x y, \quad(i=1,2,3)$, and where $C_{i}$ has the discriminant $\dagger$

$$
\Delta_{i} \equiv f_{i} g_{i} h_{i}+a_{i} f_{i}^{2}+b_{i} g_{i}^{2}+c_{i} h_{i}^{2} .
$$

Such a field is denoted for brevity by the symbol $G F\left(2^{n}\right)$. Along with (1) we consider the cubic curve in $\lambda, \mu, \nu$ that is obtained by equating to zero the discriminant of the general conic of (1) $\ddagger$

* Presented to the Society, December 31, 1926.

$\dagger$ See A. D. Campbell, Plane cubic curves in the Galois fields of order $2^{n}$, Annals of Mathematics, vol. 27 (1926), p. 395.

łCompare C. Jordan, Réduction d'un réseau de formes quadratiques, Journal des Mathématiques, (6), vol. 2 (1906), p. 412. 


$$
\begin{gathered}
K \equiv a \lambda^{3}+b \mu^{3}+c \nu^{3}+d \lambda^{2} \mu+e \lambda \mu^{2}+f \lambda^{2} \nu+g \lambda \nu^{2} \\
+h \mu^{2} \nu+j \mu \nu^{2}+k \lambda \mu \nu^{\prime}=0
\end{gathered}
$$

where

$$
a \equiv \Delta_{1}, b \equiv \Delta_{2}, c \equiv \Delta_{3}, \quad \text { and }
$$

$$
d \equiv f_{1} g_{1} h_{2}+f_{1} g_{2} h_{1}+f_{2} g_{1} h_{1}+a_{2} f_{1}{ }^{2}+b_{2} g_{1}{ }^{2}+c_{2} h_{1}{ }^{2},
$$

with similar expressions for $e, f, g, h, j$; and where

$$
k \equiv f_{1} g_{2} h_{3}+f_{1} g_{3} h_{2}+f_{2} g_{1} h_{3}+f_{2} g_{3} h_{1}+f_{3} g_{1} h_{2}+f_{3} g_{2} h_{1} .
$$

In this paper we shall classify these nets of conics, deriving a typical net for each class. First we shall study the nets that have no degenerate pencil of conics* and hence have a non-composite cubic $K=0$; then we shall consider the nets with composite cubics. For the first type of nets we reduce the cubic to a standard form and then put the net in a canonical form.

If the net (1) has a double line this must correspond to a cusp on the cubic (2), a fact easy to prove. We put (2) in the form $\dagger$

$$
\lambda^{2} \mu+\lambda^{2} \nu+\mu \nu^{2}=0
$$

with a cusp at $(0,1,0)$ and an inflection at $(1,0,0)$. To the tangent at the inflection on the cubic there must correspond in the net a pencil with just one degenerate conic $C$ which corresponds to the inflection. This conic $C$ must be a real line pair, because there is no such pencil with $C$ a pair of conjugate imaginary lines. $\ddagger$ We can now put (1) in the form

$$
\begin{gathered}
\lambda y z+\mu x^{2}+\nu C_{3}=0, \\
K \equiv \lambda^{2} \mu+a_{3} \lambda^{2} \nu+g_{3} h_{3} \lambda \nu^{2}+f_{3}^{2} \mu \nu^{2}+\Delta_{3} \nu^{3}=0 .
\end{gathered}
$$

We must have $a_{3}=1, g_{3} h_{3}=0, f_{3}^{2}=1, \Delta_{3}=0$; hence $h_{3}=0$ or $g_{3}=0$. We can assume that $h_{3}=0$, because the transformation

* That is, a pencil with $\Delta \equiv 0$.

†Campbell, loc. cit., p. 398. See this paper also for the other typical forms of cubics.

$\ddagger$ See A. D. Campbell, Pencils of conics in the Galois fields of order $2^{n}$, American Journal of Mathematics, vol. 49 (1927), pp. 401-406 for such facts and for typical pencils of conics. 
$x=x^{\prime}, y=z^{\prime}, z=y^{\prime}$ reduces the case $g_{3}=0$ to the case $h_{3}=0$. Therefore we have $h_{3}=0, c_{3} g_{3}^{2}+1=0$. If $c_{3} \neq 0$, we put*

then

$$
\begin{aligned}
& \lambda=\lambda^{\prime}+\nu^{\prime}, \quad \mu=\mu^{\prime}+\nu^{\prime}, \quad \nu=\nu^{\prime} ; \\
& x=\frac{1}{g_{3}} x^{\prime}, \quad y=g_{3} y^{\prime}, \quad z=\frac{1}{c_{3}^{1 / 2}} z^{\prime} ;
\end{aligned}
$$

$$
\lambda^{\prime}=\frac{c_{3}^{1 / 2}}{g_{3}} \lambda^{\prime \prime}, \mu^{\prime}=g_{3}^{2} \mu^{\prime \prime}, \nu^{\prime}=\nu^{\prime \prime} ;
$$

and after dropping the primes and double primes from variables and parameters we obtain

$$
\lambda y z+\mu x^{2}+\nu\left(y^{2}+z^{2}+z x\right)=0, K \equiv \lambda^{2} \mu+\nu^{3}=0,
$$

whose cubic is reducible to the above typical cubic. If $c_{3}=0$, we get

$$
\lambda y z+\mu x^{2}+\nu\left(y^{2}+z x\right)=0, K \equiv \lambda^{2} \mu+\nu^{3}=0 .
$$

The cubics of these two nets are the same, but any transformation that is to send (3) into (4) must send the pencil $\nu=0$ of (3) into the same pencil of (4) $t$ and so must have the form

or

$$
\begin{aligned}
T: \rho x & =\alpha_{1} x^{\prime}, & \rho y=\beta_{2} y^{\prime}, & \rho z=\gamma_{3} z^{\prime} ; \\
\rho x & =\alpha_{1} x^{\prime}, & \rho y=\gamma_{2} z^{\prime}, & \rho z=\beta_{3} y^{\prime} .
\end{aligned}
$$

However, $T$ sends (3) into a net containing the term in $z^{2}$, hence (3) and (4) are non-equivalent.

Next we suppose the cubic (2) to have a crunode and no real inflection, and we reduce the cubic to the form

$$
\alpha \lambda^{3}+\mu^{3}+\lambda \mu \nu=0,
$$

with crunode at $(0,0,1)$, where $\alpha \neq$ cube and $2^{n}=3 m+1$. As we discussed above the sort of conic in the net that must

* We call this change of parameters "using $C_{1}$ to rid $C_{3}$ of $y z$, and using $C_{2}$ to rid $C_{3}$ of $x^{2}$."

†Because the double line has to go into a double line, also $\nu=0$ in the $(\lambda, \mu, \nu)$ plane joins the cusp to the inflection on the cubic and so must go into the same line on the other cubic. 
correspond to an inflection on the cubic so here we can readily show that to the crunode there must correspond a real line pair in the net. We put our net in the form

$$
\begin{aligned}
\lambda C_{1}+\mu\left(y^{2}+z x\right)+\nu x_{3} & =0, K^{*} \equiv \Delta_{1} \lambda^{3}+\left(f h+g^{2}\right) \lambda^{2} \mu \\
& +b \lambda \mu^{2}+f g \lambda^{2} \nu+c \lambda \nu^{2}+f \lambda \mu \nu+\mu^{3}=0 .
\end{aligned}
$$

We must have $b=c=g=0, f=1, h=0, a=\alpha \neq$ cube; hence we get

$$
\lambda\left(\alpha x^{2}+y z\right)+\mu\left(y^{2}+z x\right)+\nu x y=0 .
$$

Now we consider the cubic (2) to have a crunode and just one real inflection, and put the cubic in the form

$$
\lambda^{3}+\mu^{2} \nu+\lambda \mu \nu=0,
$$

with crunode at $(0,0,1)$ and inflection at $(0,1,0)$, which cubic exists only in the $G F(2)$. We reduce the net to the form

$$
\begin{gathered}
\lambda\left(y^{2}+z x\right)+\mu C_{2}+\nu x y=0, \quad K \equiv \lambda^{3}+b \lambda^{2} \mu+\left(f h+g^{2}\right) \lambda \mu^{2} \\
+f \lambda \mu \nu+f g \mu^{2} \nu+c \mu \nu^{2}+\Delta_{2} \mu^{3}=0 .
\end{gathered}
$$

We have $b=0, f=g=h=a=1, c=0$. We use $C_{3}$ to rid $C_{2}$ of $x y$ and we get

$$
\lambda\left(y^{2}+z x\right)+\mu\left(x^{2}+y z+z x\right)+\nu x y=0 .
$$

If for a net (1) the cubic (2) has a crunode and at least two real inflections, we put this cubic in the form

$$
\lambda^{2} \mu+\lambda \mu^{2}+\lambda^{2} \nu+\mu^{2} \nu+\lambda \mu \nu=0,
$$

existent only in $G F\left(2^{n}\right)$ for $n>1$, with crunode at $(0,0,1)$ and inflections at $(1,0,0)$ and $(0,1,0)$. The net can be written

$$
\begin{gathered}
\lambda C_{1}+\mu x y+\nu\left(z^{2}+z x\right)=0, \quad K \equiv \Delta_{1} \lambda^{3}+f g \lambda^{2} \mu+c \lambda \mu^{2} \\
+\left(f h+h^{2}\right) \lambda^{2} \nu+b \lambda \nu^{2}+\mu^{2} \nu+f \lambda \mu \nu=0 .
\end{gathered}
$$

We find that $f g=c=f h+h^{2}=1, b=0, f=1, \Delta_{1} \equiv h+a+h^{2}=0$;

* We shall from now on omit the subscripts from such coefficients as $f_{1}, h_{1}$, etc., and $a_{2}, b_{2}$, etc., and $a_{3}, b_{3}$, etc. 
hence $g=h^{2}+h=a=1$. We use $C_{2}$ to rid $C_{1}$ of $x y$ and $C_{3}$ to rid $C_{1}$ of $z^{2}+z x$, and we obtain

$$
\lambda\left(x^{2}+y z\right)+\mu x y+\nu\left(z^{2}+z x\right)=0 .
$$

We consider the net (1) to have a cubic (2) with an acnode and no real inflections, and we reduce the cubic to the form

$$
\lambda^{2} \mu+\lambda^{2} \nu+\alpha \mu^{2} \nu+\lambda \mu \nu=0,
$$

with acnode at $(0,0,1)$ and $\lambda^{2}+\alpha \mu^{2}+\lambda \mu=0$ as tangents. If we suppose that to an acnode there corresponds a real line pair in the net we are led to a contradiction, hence to the acnode there must correspond a conjugate imaginary line pair. Therefore we can put (1) in the form

$$
\begin{gathered}
\lambda C_{1}+\mu x y+\nu\left(x^{2}+z^{2}+\alpha z x\right)=0, \\
K \equiv \Delta_{1} \lambda^{3}+f g \lambda^{2} \mu+c \lambda \mu^{2}+\left(h^{2}+f^{2}+\alpha f h\right) \lambda^{2} \nu \\
+\alpha^{2} b \lambda \nu^{2}+\mu^{2} \nu+\alpha f \lambda \mu \nu=0 .
\end{gathered}
$$

We must have $c=b=\Delta_{1}=0, f g=\alpha f=h^{2}+f^{2}+\alpha f h$; hence $g=\alpha, f g h+a f^{2} \equiv \alpha f h+a f^{2}=0$. We use $C_{2}$ to rid $C_{1}$ of $x y$, and put

$$
\lambda=\frac{f}{\alpha h} \lambda^{\prime}, \quad \mu=\frac{f^{2}}{\alpha h} \mu^{\prime}, \nu=\nu^{\prime} ; x=x^{\prime}, y=\frac{\alpha h}{f^{2}} y^{\prime}, z=z^{\prime}
$$

and we get

(8) $\lambda\left(x^{2}+y z+f z x / h\right)+\mu x y+\nu\left(x^{2}+z^{2}+\alpha z x\right)=0$,

where $h^{2}+f^{2}+\alpha f h+\alpha f=0$.

We deal with a net whose cubic has an acnode and just one real inflection, and we put this cubic in the form

$$
\lambda^{3}+\lambda^{2} \nu+\alpha \mu^{2} \nu+\lambda \mu \nu=0,
$$

which is nonexistent in $G F(2)$, with acnode at $(0,0,1)$ and inflection at $(0,1,0)$. We can put the net in the above form $(n)$ with its cubic $K=0$. Hence we see that $f g=b=c=0$, $\alpha f=h^{2}+f^{2}+\alpha f h=\Delta_{1} \neq 0$; therefore $g=0, f \neq 0, a f^{2}=\alpha f, a=\alpha / f$. We use $C_{2}$ to rid $C_{1}$ of $x y$, then put 


$$
\lambda=\frac{f}{\alpha} \lambda^{\prime}, \mu=\frac{f^{2}}{\alpha} \mu_{1}^{\prime}, \nu=\nu^{\prime} ; x=x^{\prime}, y=\frac{\alpha}{f^{2}} y^{\prime}, z=z^{\prime}
$$

and we obtain

$$
\lambda\left(x^{2}+y z\right)+\mu x y+\nu\left(x^{2}+z^{2}+\alpha z x\right)=0 .
$$

Next we study the net (1) when it has a cubic (2) with an acnode and at least two real inflections, and we reduce this cubic to the form

$$
\lambda^{2} \mu+\lambda \mu^{2}+\lambda^{2} \nu+\mu^{2} \nu+\lambda \mu \nu=0,
$$

existent only in $G F(2)$, with acnode at $(0,0,1)$ and one real inflection at $(0,1,0)$. We can put our net in the above form $(n)$ with $\alpha=1$. We must have $f g=c=h^{2}+f^{2}+f h=f=1$, $b=0, \Delta_{1}=0$; hence $f=g=c=1, h=1$ and $a=0$, or $h=0$ and $a=0$. We use $C_{2}$ to rid $C_{1}$ of $x y$ and we get

$$
\lambda\left(z^{2}+y z+z x\right)+\mu x y+\nu\left(x^{2}+z^{2}+z x\right)=0 .
$$

Now we assume that the net (1) has a cubic (2) with no node or cusp and no real inflection, and we put this cubic in the form

$$
\lambda^{2} \mu+\lambda \mu^{2}+\alpha \lambda \nu^{2}+\beta \mu \nu^{2}+\lambda \mu \nu=0 .
$$

The point $(0,1,0)$ on this cubic was any point on the cubic, so we take a point that corresponds to a real line pair in the net, and put our net in the form

$$
\begin{gathered}
\lambda C_{1}+\mu\left(z^{2}+z x\right)+\nu x y=0, \quad K \equiv \Delta_{1} \lambda^{3}+\left(f h+h^{2}\right) \lambda^{2} \mu \\
+b \lambda \mu^{2}+f g \lambda^{2} \nu+c \lambda \nu^{2}+\mu \nu^{2}+f \lambda \mu \nu=0 .
\end{gathered}
$$

We see we must have $\Delta_{1}=0, f \neq 0, f g=0, b=f h+h^{2}=f \neq 0$; hence $g=0, \Delta_{1} \equiv a f^{2}+c h^{2}=0$. We can therefore reduce our net to the form

$$
\begin{aligned}
\lambda\left[\frac{c\left(h^{2}+1\right)(h+1)}{h^{4}} x^{2}+y^{2}\right. & \left.+\frac{c(h+1)}{h^{2}} z^{2}+y z\right] \\
& +\mu\left(z^{2}+z x\right)+\nu x y=0,
\end{aligned}
$$

with a cubic that is transformable in to the above typical cubic with $\alpha=c(h+1) / h^{2}, \beta=(h+1) / h^{2}$. 
We consider the net (1) when its cubic (2) has no node or cusp and just one real inflection and can be put in the form

$$
\lambda^{2} \mu+\lambda \mu^{2}+\alpha \lambda \nu^{2}+\mu^{2} \nu+\lambda \mu \nu=0,
$$

with inflection at $(0,1,0)$. Since there are two real tangents to the cubic from the point $(0,0,1)$, this point must correspond to a real line pair in the net.* We can reduce the net to the above form $(n)$. We have then $f g=c=1, f h+h^{2}=0$, $b=\alpha, f=1, \Delta_{1}=0$; hence $h=1$ or $h=0, g=1, a=\alpha$. We use $C_{2}$ to rid $C_{1}$ of $x y$ and get

$$
\lambda\left(\alpha x^{2}+\alpha y^{2}+z^{2}+y z+z x\right)+\mu x y+\nu\left(z^{2}+z x\right)=0 .
$$

We suppose the cubic (2) has no node or cusp but just one real inflection and can be put in the form

$$
\beta \lambda^{3}+\lambda^{2} \nu+\lambda \nu^{2}+\alpha \mu^{2} \nu+\lambda \mu \nu=0 .
$$

We reduce the net (1) to the form

$$
\begin{gathered}
\lambda\left(y^{2}+z x\right)+\mu x y+\nu C_{3}=0, \quad K \equiv \lambda^{3}+b \lambda^{2} \nu+\left(f h+g^{2}\right) \lambda \nu^{2} \\
+c \mu^{2} \nu+f g \mu \nu^{2}+f \lambda \mu \nu+\Delta_{3} \nu^{3}=0 .
\end{gathered}
$$

This gives us $b=f h+g^{2}=f \neq 0, f g=0, \Delta_{3}=0$; hence $g=0, h=1$, $a=c / f^{2}$. By an obvious transformation on $\lambda, \mu, \nu$ alone, we get

$$
\lambda\left(y^{2}+z x\right)+\mu x y+\nu\left(\frac{c}{f^{3}} x^{2}+y^{2}+\frac{c}{f} z^{2}+y z\right)=0,
$$

with cubic reducible to the above typical form with $\alpha=c / f$, $\beta=1 / f$.

Next we consider the net (1) when its cubic (2) has no node or cusp but at least two real inflections, and reduce this cubic to the form

$$
\alpha \nu^{3}+\lambda^{2} \mu+\lambda \mu^{2}+\lambda \mu \nu=0,
$$

with inflections at $(0,1,0)$ and $(1,0,0)$. We put our net in the form

* This fact can be observed by noting the pencils of conics in the $G F\left(2^{n}\right)$ that have discriminants of the form $\lambda^{2} \mu$. 


$$
\begin{array}{r}
\lambda C_{1}+\mu x y+\nu\left(y^{2}+z x\right)=0, \quad K \equiv \Delta_{1} \lambda^{3}+f g \lambda^{2} \mu+c \lambda \mu^{2} \\
+\left(f h+g^{2}\right) \lambda^{2} \nu+b \lambda \nu^{2}+\nu^{3}+f \lambda \mu \nu=0 .
\end{array}
$$

We must have $f g=c=f \neq 0, f h+g^{2}=b=\Delta_{1}=0$; hence $g=1$, $c=f, h=1 / f, a=(f+1) / f^{3}$. Using $C_{2}$ to rid $C_{1}$ of $x y$ we get

$$
\lambda\left(\frac{f+1}{f^{3}} x^{2}+f z^{2}+f y z+z x\right)+\mu x y+\nu\left(y^{2}+z x\right)=0,
$$

with cubic reducible to the above type form with $\alpha=1 / f$.

Finally we suppose our net (1) has no degenerate conic and therefore its cubic (2) is imaginary. We put our cubic in either one of the two forms

$$
\lambda^{3}+\alpha^{\prime} \mu^{3}+\beta^{\prime} \nu^{3}+\gamma^{\prime} \lambda \nu^{2}+\mu \nu^{2}+\lambda \mu \nu=0
$$

or

$$
\lambda^{3}+\alpha^{\prime} \mu^{3}+\beta^{\prime} \nu^{3}+\lambda \mu \nu=0,
$$

where $\alpha^{\prime}$ and $\beta^{\prime}$ are non-cubes and $2^{n}=3 m+1$. Then the net (1) can be put in the form

$$
\begin{gathered}
\lambda\left(z^{2}+x y\right)+\mu\left(\alpha x^{2}+\beta y^{2}+y z\right)+\nu C_{3}=0, \\
K \equiv \lambda^{3}+\alpha \mu^{3}+c \lambda^{2} \nu+\left(f g+h^{2}\right) \lambda \nu^{2}+\alpha \mu^{2} \nu \\
\quad+\left(g h+\alpha f^{2}+\beta g^{2}\right) \mu \nu^{2}+g \lambda \mu \nu+\Delta_{3} \nu^{3}=0 .
\end{gathered}
$$

We must have $c=f g+h^{2}=0, g \neq 0$. We put

and we get

$$
x=\frac{1}{g} x^{\prime}+\frac{f}{g} y^{\prime}, \quad y=y^{\prime}, z=\frac{h}{g} y^{\prime}+z^{\prime},
$$

$$
\begin{aligned}
\lambda\left(z^{2}+b_{1} y^{2}+h_{1} x y\right)+\mu\left(a_{2} x^{2}+b_{2} y^{2}\right. & \left.+f_{2} x z\right) \\
& +\nu\left(a_{3} x^{2}+b_{3} y^{2}+z x\right)=0, \\
K \equiv h_{1}^{2} \lambda^{3}+b_{1} \lambda \nu^{2}+a_{2} f_{2}^{2} \mu^{3}+a_{3} f_{2}^{2} \mu^{2} \nu & +b_{2} \mu^{2} \\
& +b_{3} \nu^{3}+f_{2} h_{1} \lambda \mu \nu=0 .
\end{aligned}
$$

This cubic must have one of the above forms, hence we see that $h_{1}^{2}=f_{2} h_{1} \neq 0, a_{3}=0$, so $f_{2}=h_{1} \neq 0$, and we get

(15) $\lambda\left(z^{2}+\gamma y^{2}+x y\right)+\mu\left(\alpha x^{2}+\delta y^{2}+y z\right)+\nu\left(\beta y^{2}+z x\right)=0$, where $\alpha \beta \neq 0, \alpha$ and $\beta$ non-cubes, $\gamma$ may be zero, $\delta=1$ or 0 . 
We shall not derive the nets with degenerate cubics but merely list them below, because their derivation is so simple by the method employed by C. Jordan, loc. cit.

(21) $\lambda x^{2}+\mu x y+\nu\left(z^{2}+y z\right)=0$.

(22) $\lambda x^{2}+\mu x y+\nu y z=0$.

(23) $\lambda x^{2}+\mu x y+\nu\left(y^{2}+z^{2}+\alpha y z\right)=0$, $y^{2}+z^{2}+\alpha y z=0$ irreducible.

(24) $\lambda x^{2}+\mu x y+\nu\left(z^{2}+z x\right)=0$.

(25) $\lambda x^{2}+\mu x y+\nu\left(y^{2}+z x\right)=0$.

(26) $\lambda x^{2}+\mu x y+\nu x z=0$.

(27) $\lambda x^{2}+\mu\left(y^{2}+x y\right)+\nu\left(z^{2}+y z\right)=0$.

(28) $\lambda x^{2}+\mu\left(y^{2}+x y\right)+\nu y z=0$.

(29) $\lambda x^{2}+\mu\left(y^{2}+x y\right)+v\left(\alpha y^{2}+z^{2}+z x\right)=0$, $\mu^{2}+\alpha \nu^{2}+\mu \nu=0$ irreducible.

(30) $\lambda x^{2}+\mu\left(y^{2}+x y\right)+\nu\left(z^{2}+z x\right)=0$.

(31) $\quad \lambda x y+\mu x z+\nu\left(x^{2}+y^{2}+z^{2}+\alpha y z\right)=0$.

(32) $\quad \lambda x y+\mu x z+\nu\left(y^{2}+z^{2}+\alpha y z\right)=0$.

(33) $\quad \lambda x y+\mu x z+\nu\left(x^{2}+y z\right)=0$.

(34) $\quad \lambda x y+\mu x z+\nu y z=0$.

\section{Syracuse UnIVERsity}

